Departamento de Historia

Universidad de Santiago de Chile

Revista de Historia Social

y de las Mentalidades

Volumen 24, $\mathrm{N}^{\circ}$ 1, 2020: 85-108

Issn On Line: 0719-4749

\title{
REFLEXIONES SOBRE LA ENTREVISTA COMO DOCUMENTO HISTÓRICO Y MATERIAL DE ARCHIVO*
}

\author{
REFLECTIONS ON THE INTERVIEW AS A \\ HISTORICAL DOCUMENT AND ARCHIVE MATERIAL
}

\author{
DRA. JACQUELINE DUSSAILLANT CHRISTIE** \\ CIDOC- Universidad Finis Terrae \\ Santiago, Chile \\ Email: jdussaillant@uft.cl \\ Id ORCID: 0000-0001-5712-0108
}

\begin{abstract}
RESUMEN
La entrevista ha sido utilizada para levantar información por investigadores de diversas disciplinas y empleando para ello métodos y tecnologías distintas. Este artículo revisa los principales problemas asociados a la entrevista en el contexto del surgimiento de la historia oral, tanto desde la perspectiva de su creación y conservación, como de su uso cuando es integrada a un archivo de acceso público. Concluye que, si en un comienzo los principales desafíos para archiveros e historiadores radicaban en la metodología y pertinencia de la entrevista como medio y producto, en las últimas décadas se han concentrado en aspectos éticos y legales.
\end{abstract}

Palabras clave: Entrevista; historia oral; archivo; propiedad intelectual

\begin{abstract}
The interview has been used to gather information by researchers from different disciplines and using different methods and technologies. This article reviews the main problems associated with the interview in the context of the emergence of oral history, both from the perspective of its creation and conservation, and its use when it is integrated into a public access Archive. It concludes that if at the beginning the main challenges for archivists and historians lay in the methodology and relevance of the interview as a means and product, in the last decades they have concentrated on ethical and legal aspects.
\end{abstract}

Keywords: Interview; Oral History; Archive; Intellectual Property

* $\quad$ Recibido: 20 de diciembre de 2019; Aprobado: 24 de enero de 2020.

** Artículo de revisión. Este artículo surge de un trabajo presentado para el Seminario Archivo organizado por el Centro de Investigación y Documentación (CIDOC) de la Universidad Finis Terrae, en el 2018. 
Cómo citar: Dussaillant Ch., Jacqueline. (2020). "Reflexiones sobre la entrevista como documento histórico y material de archivo". Revista Historia Social y de las Mentalidades, 24(1), 85-108. DOI: 10.35588/rhsm.v24i1.4285.

\section{INTRODUCCIÓN}

Un proverbio africano que dice que "cada vez que un anciano muere, una biblioteca se quema" (Perks y Thomson xiii) adquiere pleno sentido en el contexto del desarrollo de lo que en la actualidad conocemos como historia oral, en especial si consideramos que esta se nutre básicamente de la entrevista. En su libro The Voice of the Past. Oral History, Paul Thompson explicaba en 1978 que la historia oral había cambiado tanto el contenido de la historia, como el proceso de escribirla (Thompson 3-7). Que de los nueve capítulos haya destinado uno a la entrevista es natural, pero lo que es destacable es que también haya dedicado otro al problema de su almacenamiento y administración; poniendo así en evidencia lo relevante que resulta lo que podría considerarse un "efecto colateral" de la historia oral, a saber, los desafíos para su conservación y, en especial, el destino final del material recogido y su uso por otros investigadores. Sin duda, estos últimos aspectos forman también parte esencial del quehacer de cualquier investigador que trabaje con entrevistas $y$, con mayor razón para el caso del historiador, acostumbrado y dependiente del trabajo en archivos. Este no puede ignorar que, al entrevistar a alguien para recabar información sobre el pasado reciente, está también creando un documento que eventualmente será consultado tanto por los colegas de su generación como de las futuras.

Cuando en la década de 1960 los historiadores experimentaron con la historia oral como metodología de trabajo, tendieron a adoptar una postura defensiva en relación con la objetividad, veracidad y confiabilidad de la memoria sobre la cual esta descansa, que parecían ser sus principales desafíos (Thompson 25 y ss.; Charlton et al. 227). Desde entonces, utilizaron herramientas interdisciplinarias tomadas de diferentes áreas del conocimiento, desde la crítica literaria y la narrativa, hasta la etnografía y el folclore. Todo ello se tradujo en que, a lo largo de las décadas siguientes, las fuentes orales fueron objeto de suficientes trabajos teóricos y metodológicos que, más allá de dar cuenta de la "oportunidad única" que representaban en términos epistemológicos (Barela et al. 8), abordaron la necesidad de mantener ciertos resguardos en términos archivísticos, legales y éticos (Samuel, 1972; Lance, 1978; Stielow, 1986; Neuenschwander, 1993; Ward, 1995; Lawrence, 2016).

La revisión de estos y otros autores permite rescatar algunos de los puntos que han estado en discusión a medida que la entrevista fue ganando más adeptos 
entre investigadores de diversas disciplinas, entre ellas, la historia. ¿Por qué incorporar en un archivo las palabras subjetivas de los actores de la historia?, ¿Sus olvidos, reconstrucciones y emociones no influyen negativamente en la percepción de los documentos? ¿Documentos y memorias no pertenecen a dos temporalidades heterogéneas e irreconciliables? ¿De qué modo y por cuánto tiempo debe resguardarse la privacidad de los entrevistados? ¿La memoria de las personas atrapada en el tiempo de su oralidad, son para el historiador un archivo como cualquier otro? Aunque responder a estas preguntas excede a los objetivos de este artículo, sirven para dar cuenta de las múltiples aristas y puntos de discusión que han sido abordados no solo por los propios investigadores, sino también por aquellos especialistas destinados a resguardar las entrevistas en cuanto documento, los archiveros.

Este artículo tiene dos propósitos más acotados. El primero, dar cuenta de los principales aspectos que se han debatido en relación con la trilogía entrevista-historiador-archivo desde los primeros intentos por hacer historia oral desde fines de la década de 1950 hasta nuestros días. El segundo, y derivado del anterior, es abordar fundamentalmente los problemas metodológicos, éticos y legales derivados de la discusión acerca de a quién "pertenece" la voz registrada en una entrevista y, consecuentemente, cómo transmitirla al público y a futuras generaciones. Esta nace normalmente con un propósito específico, de manera que su creación reviste un carácter muy particular, si no personal. Luego, su registro - en cualquiera de sus formatos- o bien queda guardado en la gaveta del investigador original -expuesto así al olvido o a su eventual pérdida o deterioroo bien pasa a ser parte de un archivo para su empleo por otros investigadores. Tanto la gaveta personal como el archivo público, implican ventajas y desventajas.

Teniendo en cuenta que la razón de ser de cualquier archivo radica en su uso, el caso de las entrevistas reviste una serie de complejidades e interrogantes que pretendemos relevar. Para ello, además de la revisión de una selección bibliográfica sobre el tema en cuestión, hemos escogido la revista The American Archivist como principal marco de referencia. Esta publicación nació en 1938 para dar voz a la Society of American Archivists, con el objetivo de "ser lo más útil posible a los miembros de la profesión [archivística]" (I 1v). Debido a su larga duración, tiene la ventaja que permite observar en el tiempo el surgimiento de la preocupación de la entrevista como fuente y los problemas y desafíos que década tras década fueron aquejando a archivistas, bibliotecarios e investigadores, en su mayoría historiadores interesados en la historia oral. Observamos que muchos de los temas abordados por sus múltiples autores responden a cuestiones circunstanciales, como aquellos derivados de la tecnología asociada a la entrevista; mientras que otros atraviesan gran parte del periodo estudiado. Hemos organizado este artículo en tres partes. La 
primera revisa algunos antecedentes relevantes acerca de las primeras experiencias dadas en la relación entre entrevista, historia y memoria. La segunda, da cuenta de los problemas y desafíos observados por archiveros e historiadores dedicados a la historia oral sobre el tema en cuestión entre 1950 y 2019 a través de la revista The American Archivist. Por último, terminamos abordando algunas de las derivadas legales y éticas que vinculan a la entrevista como documento de archivo para el uso público.

\section{LA ENTREVISTA Y LA MEMORIA, ALGUNAS EXPERIENCIAS}

La entrevista, como método para recabar información, tiene larguísima data y difícilmente se podría precisar su origen. De hecho, Heródoto y en especial Tucídides son reconocidos por haber recurrido a ella para obtener información de primera mano. Pero, la entrevista empleada de manera sistemática y con el fin de ser conservada para utilizarla con fines patrimoniales y científicos, tiene comienzos mejor definidos. Algunos se remontan a la década de 1920, cuando un grupo de antropólogos de la Universidad de Chicago hizo entrevistas biográficas tanto a campesinos como obreros y a sus familias, en el contexto de una ciudad que empezaba a ver los problemas asociados a las grandes industrias, las protestas sociales y los conflictos urbanos. ${ }^{1} \mathrm{Al}$ respecto, el referente indiscutido es el trabajo titulado The Polish Peasant: in Europe and in America (1918-1920) en el que William I. Thomas estudió el problema de la inmigración tomando el caso de campesinos polacos en Polonia y en Chicago en especial a través de documentos personales, tales como cartas y diarios de vida (Santos 60).

Con este tipo de experiencias, la escuela sociológica de dicha universidad se transformó en un centro relevante desde la perspectiva metodológica en las primeras décadas del siglo XX. Esto se explica en parte en el rol innovador desarrollado en la investigación empírica, la creación de novedosas estrategias de recolección de datos, pero también por el acento puesto en "la producción de información de primera mano" (Santos 58). Según Javier Santos, aunque no se tratara de métodos del todo nuevos, es precisamente el empleo de documentos personales como fuentes, sumado al énfasis en el trabajo de campo directo, lo que le valió a dicha escuela "un liderazgo indiscutido en la investigación empírica hasta mediados de la década de 1930" (58):

1 Un libro que trata en profundidad esta experiencia es el de Jean-Michel Chapoulie, La tradition sociologique de Chicago (1892-1961), Seuil, 2001. 
Todas las investigaciones realizadas por los miembros de la Escuela de Chicago se apoyaron en mayor o menor medida en información documental que incluía documentación no personal (como registros públicos, de asociaciones de asistencia social, de iglesias, censos, entre otros) y varios tipos de documentación personal (principalmente en la forma de autobiografías, diarios íntimos y cartas personales). Sin embargo, estas fuentes se articulaban, las más de las veces, con el desarrollo de trabajo de campo (entrevistas formales o informales y observaciones con distintos grados de participación). Esto último tenía como finalidad el acceso en directo al fenómeno social abordado, y muchas veces permitía construir los corpus de documentos personales a analizar en el marco de las investigaciones. (59) ${ }^{2}$

Además de los avances dados en el uso del testimonio oral para el levantamiento de datos y la construcción de la memoria al alero de instituciones académicas, otras experiencias similares contribuyeron a la consolidación de un método y a la puesta en valor de sus resultados. En este sentido, puede afirmarse que la decisión de recoger la memoria de los combatientes en la Segunda Guerra Mundial, tras un llamado hecho con dicho propósito por Franklin D. Roosevelt, fue sin duda un hito de gran relevancia en la vinculación entre la entrevista sistemática y la voluntad política de guardar parte de la historia del tiempo presente en un archivo. Esas primeras entrevistas fueron hechas al calor de la guerra, en noviembre de 1943. Al final de dicho conflicto, se habían recogido unas 2.000 entrevistas a soldados, algunos en calidad de prisioneros de guerra. Dichas voces recibieron pronto, de parte de la historiadora Georgette Elgey, el nombre de "testimonios inmediatos", pues casi nunca fueron recogidos más allá de diez días tras los hechos (Duclert 70). La experiencia no tardó en institucionalizarse y ser replicada tanto a nivel de iniciativas estatales como académicas. Uno de los casos más reconocidos fue el que lideró el periodista del New York Times Allan Nevins, quien, tras ingresar a enseñar en la Universidad de Columbia, creó la "Columbia Oral History Office" en 1948. En la actualidad, dicho archivo cuenta con más de diez mil entrevistas, siendo una de las más grandes de su tipo en Estados Unidos. A partir de entonces, se hicieron una serie de entrevistas a actores de la vida política, económica, científica, intelectual, artística, religiosa; impulsadas y recogidas por diferentes instituciones, como museos, bibliotecas y especialmente universidades (Duclert 72).

2 La cursiva es nuestra. 
Al otro lado del Atlántico también se cuenta con experiencias similares. Entre ellas destaca la Comisión de Historia de la Ocupación y de la Liberación de Francia que fue constituida en octubre de 1944 pero que en 1951 fue reemplazada por el Comité de Historia de la Segunda Guerra Mundial. Este organismo adjunto a la Presidencia del Consejo, estaba compuesto por representantes de once ministerios, algunos servicios que poseían archivos (como Services Historiques des Armées y Archives de France, entre otros) y algunas bibliotecas. Inició sus actividades bajo la dirección de historiadores, entre estos, Lucien Febvre como presidente y Henri Michel como secretario general. A ellos se sumaron también algunos sociólogos y juristas en calidad de colaboradores. Como el propio Michel lo señaló en un artículo que relata la experiencia del Comité, el equipo tenía la inédita misión de realizar una investigación histórica que, pese a surgir de una iniciativa vinculada a organismos gubernamentales, contaba con plena libertad, pues no pretendía crear una "historia oficial" (Michel 3). Entre la enorme documentación recogida durante varios años, que incluye cartas, fotografías y periódicos, destacan las encuestas y entrevistas. Estas permitieron realizar, en palabras de Michel, el primer intento efectuado de manera pragmática del método bautizado más tarde como "historia oral" (Michel 7). Este comité llevó a cabo verdaderas campañas de entrevistas hasta alcanzar a recoger unos 3.500 registros.

A estas experiencias oficiales de gran escala se agregaron muchas otras de carácter local. En consecuencia, para la década de 1970 los historiadores franceses ya habían descubierto el valor de la entrevista como método para recoger información, y la empleaban para cubrir diferentes hechos de la historia contemporánea y ya no solo, como fue en su origen, en relación con el conflicto armado. En esa lógica nació hacia 1978 el Instituto de Historia del Tiempo Presente (Duclert 74). Este instituto, además, impulsó una reflexión teórica sobre las prácticas de la dimensión oral de la historia, a través de coloquios, charlas, y otras actividades. Buscó, según Duclert, diferenciarse de la experiencia anglosajona, a través del acercamiento con las ciencias sociales, el encuentro entre los pioneros de la historia oral y las nuevas generaciones de investigadores, y mediante el apoyo al trabajo de los archivistas (74).

En los años noventa, otra experiencia histórica particular dio origen a una iniciativa europea que merece ser considerada: la del archivo "Memoria alemana". Los investigadores Lutz Niethammer y Alexander Von Plato habían hecho entrevistas a gran escala entre cientos de alemanes del este y oeste tras la caída del muro de Berlín. Surgió entonces la idea de almacenar dicho material, consistente en alrededor de 1.500 entrevistas. Finalmente, este proyecto se concretó como archivo "Memoria alemana" dentro del Instituto de Historia y Biografía, fundado en 1990 en la Fernuniversität Hagen (Leh, "Problems..."). 
Desde entonces este archivo se ha ido acrecentando, al sumar a las cintas de audio y sus transcripciones, una serie de otros documentos personales, tales como fotografías, diarios, cartas y otras fuentes escritas. Sin embargo, las entrevistas siguen siendo el núcleo central del mismo.

Este tipo de experiencias también se ha hecho realidad en Latinoamérica. Un ejemplo se halla en el Instituto Histórico de Buenos Aires, que comenzó a dar los primeros pasos en la historia oral en 1985 con la preparación de talleres o entrevistas colectivas en diferentes barrios de la ciudad. Esta iniciativa había sido inspirada en parte por un ejercicio de entrevistas realizadas a ancianos del Hogar Rawson que se denominó "Los abuelos cuentan la historia" (Barela et al. 10). A partir de aquel momento, los historiadores de dicho Instituto empezaron a documentarse acerca de la historia oral en términos teóricos y metodológicos, y al mismo tiempo, se dedicaron a recoger información mediante entrevistas a los residentes de algunos barrios bonaerenses. Dos años más tarde publicaron el fruto de dicho trabajo, un libro titulado Barrio y Memoria (Barela 11) y desde entonces han realizado diversos encuentros sobre historia oral. Mediante estas y otras experiencias y proyectos, este archivo ha logrado recoger alrededor de 1.100 horas de audio - algunas transcritas- y 200 horas de filmaciones, que se han ordenado de acuerdo a criterios archivísticos que no necesariamente se tenían en cuenta en décadas anteriores, pues incluyen datos del entrevistador, el entrevistado y el contexto (Barela et al. 56).

En Chile también se cuenta con algunos ejemplos de esta índole. Uno de ellos lo constituye el proyecto denominado "Cien Entrevistas", que es una colección audiovisual que alberga el Museo de la Memoria y los Derechos Humanos. Se trata de entrevistas periodísticas realizadas "a cien personajes del mundo de los derechos humanos, la política, los medios de comunicación y las artes nacionales, quienes entregan sus impresiones sobre nuestra historia reciente". ${ }^{3}$ Algo similar, a nivel regional, es el proyecto "Archivos de la Memoria de Chile", realizado entre los años 2011 y 2014, y que registró cerca de 50 entrevistas audiovisuales (Fuentealba 26). Cabe destacar la labor del historiador Gabriel Salazar en la creación de fuentes para la historia oral al relevar al sujeto popular (Nicholls 275). Al respecto, Nancy Nicholls señala que, en su origen, la historia oral en Chile se dio con experiencias de entrevistas a grupos de pobladores en el contexto de la dictadura (273). Otra iniciativa de realizar entrevistas para colaborar a la historia del Tiempo Presente es el desplegado por el Centro de Investigación y Documentación (CIDOC) de la Universidad Finis 
Terrae. Desde su creación en 1992, este centro ha realizado casi 500 entrevistas a políticos, economistas, dirigentes gremiales, autoridades de la Iglesia católica chilena, miembros de las Fuerzas Armadas, y otros actores relevantes de la historia reciente de Chile. En este caso, los contenidos, metodologías, contextos y recursos tecnológicos de las mismas son diferentes; por lo que los formatos de conservación también son distintos. Algunas de ellas fueron hechas de manera colectiva o del tipo "conversación" liderada por alguno de sus integrantes; mientras que otras estuvieron a cargo de historiadores y focalizadas en una persona en particular.

Si bien gracias a estas y otras iniciativas se ha ganado suficiente experiencia acerca de la entrevista tanto en sus facetas metodológica como archivística, el paso del tiempo y la aparición de nuevos recursos tecnológicos, como el mundo digital e Internet, siguen planteando preguntas y sumando nuevos desafíos. Como ocurre en muchos archivos que contienen entrevistas, se hace necesario reflexionar acerca de las múltiples interrogantes que presenta el testimonio oral no solo desde la perspectiva archivística, sino también respecto de su uso por parte del investigador.

\section{ENTREVISTA E HISTORIA ORAL A TRAVÉS DE THE AMERICAN ARCHIVIST}

A través de los artículos publicados en The American Archivist entre la década de 1950 y la actualidad, se observan al menos cuatro cambios importantes en la manera de percibir la entrevista. Primero, una creciente diferenciación entre la historia oral como producto y medio, lo que es clave para comprender que la entrevista no es un producto historiográfico en sí, sino que un medio con el cual trabaja el historiador. Luego, y en relación con lo anterior, se advierte un "traspaso" de responsabilidades entre archiveros e historiadores. Es decir, si inicialmente se consideraba que las entrevistas debían ser hechas por quienes estaban a cargo de los archivos con el fin de llenar vacíos de las colecciones incompletas; luego, en especial desde la década 1990, los mismos archiveros vieron la necesidad de trabajar en conjunto con los investigadores, en especial con los historiadores. Tercero, un cambio acerca de lo que era o no considerado meritorio de ser "registrado" para los futuros historiadores, y del modo en que ello debía hacerse. Cuarto, las transformaciones tecnológicas experimentadas entre 1950 y nuestros días impactó de tal manera la forma de entrevistar y de conservar y administrar dicho material, que surgieron con fuerza nuevas aristas que al comienzo no eran relevantes, en especial aquellas asociadas a cuestiones éticas y legales. 
El primer artículo en tratar el tema fue escrito en 1955 por el historiador Vaughn Davis Bornet, quien buscaba trazar una diferencia entre una entrevista común e historia oral. A su entender, esta última era un "recuerdo hablado que ha sido registrado con la ayuda de un entrevistador-historiador capacitado de acuerdo con estándares éticos y procedimentales reconocidos y escrito a máquina bajo su supervisión" (Bornet 241). En otras palabras, Bornet consideraba que, sin tales estándares y reduciéndolos solo a preguntas, respuestas, grabación electrónica y transcripción, se trataba solo de una entrevista (241). Este esfuerzo por definir el concepto de historia oral y de distinguirlo de los medios utilizados en ella, no solo continuó siendo tratado en esos años cincuenta, sino que continuó en las décadas posteriores, incluso hasta los noventa. Así, Corinne Lathrop escribió en 1957 que la entrevista de la historia oral debía diferenciarse de las encuestas de opinión pública, pues la primera debía ser "intensiva y personal e inevitablemente, con un cierto grado biográfica" (Lathrop 338). Por su parte, a mediados de la década siguiente, Gould Colman observaba que entre sus contemporáneos aún existían percepciones diferentes acerca de lo que debía ser o no ser considerada historia oral, como también acerca del valor que debía ponerse sobre los medios para abordarla. En otras palabras, acusaba que mientras algunos observaban que era aquello que se recogía con las anotaciones que hacía un historiador mientras entrevistaba a alguien de su interés para un proyecto en particular; para otros era la transcripción textual de dicha entrevista e incluso la grabación misma (Colman 79 y 80). Creía necesario aclarar el significado de "historia oral" por estimar que "está aquí para quedarse" (Colman 79). En sus palabras, "El mayor problema con la historia oral es cómo evitar confundir al investigador acerca de cuál de las tres partes involucradas en crear una transcripción -entrevistador, persona entrevistada y transcriptor- contribuyen al producto final" (80).

El énfasis puesto en la transcripción acentuaba la ambigüedad sobre el concepto de historia oral y su confusión con la entrevista en sí misma, su grabación electrónica y su supuesto producto final, la transcripción. Además, dejaba al historiador en parte al margen de la creación de la entrevista, pues no era él necesariamente quien llevaba a cabo la última etapa. En consecuencia, y dependiendo de dónde estuviera puesto el acento, en la preparación de las preguntas, su realización o su transcripción, el célebre proyecto encabezado por Allan Nevins en 1948 en la Universidad de Columbia, bien podía no ser considerado "ni oral ni historia" (Colman 80). De hecho, cabe destacar que Colman fue una de las primeras voces que llamó a conservar las cintas originales. Esto, que hoy nos parece axiomático y es lo que los archivos buscan atesorar, no era evidente en esos años. 
Esto en parte se explica porque grabar en cintas era costoso en términos económicos, por lo que incluso se solían reutilizar las cintas para posteriores entrevistas, borrando así registro tras registro. Pero, la otra razón es de orden más bien epistemológico, ya que existía una cierta tendencia a valorar más el producto en papel -la entrevista transcrita- como pieza de valor archivístico, probablemente porque se vivía todavía en lo que podríamos considerar una cultura "del papel". Por ello, lo que preocupaba a Colman no era conservar la grabación original por ser la verdadera fuente. Su énfasis en la conservación era para velar que la transcripción reprodujera exactamente lo que había dicho oralmente el entrevistado. Tal responsabilidad, dicho sea de paso, la hacía recaer sobre el entrevistador pues, dadas las limitaciones de la tecnología de la época, se consideraba natural que, por ejemplo, la entrevista se interrumpiera para cambiar de cinta, o que el aparato electrónico empleado debiera apagarse varias veces, lo que generaba posiblemente que parte de la conversación quedara fuera de la grabación (Colman 80). Donald Swain, de hecho, incluso recomendaba no grabar una entrevista si no era estrictamente necesario, ya que el entrevistado podía ser menos natural, cohibirse, y perderse así parte relevante de la información que pudiera aportar (D. Swain 65). Swain también veía como desventajas de la grabación el hecho de tener que transportar aparatos pesados y complejos, y contar con financiamiento, ya que al precio de los equipos de grabación había que sumar los costos de edición, transcripción, y otros (66). En el mismo número de la revista, sin embargo, el autor de otro artículo argumentaba que el historiador-entrevistador debía hacer la entrevista siempre premunido de una grabadora (Benison 73). Este último consideraba que la entrevista profunda era una de las etapas del trabajo, pues luego era necesario procesar y comenzar un proceso que podía tomar uno o dos años hasta completar, por ejemplo, una biografía (73).

En la medida en que se fue distinguiendo la historia oral de la entrevista, esta última aparece no solo con mayor claridad como una fuente para el historiador, sino que empieza a diferenciarse de otras fuentes orales. En 1977, por ejemplo, William W. Moss escribió que para evaluar adecuadamente los resultados que hasta entonces se había tenido con diversos proyectos sobre "historia oral", era necesario tener en consideración la existencia de tres tipos de documentación oral (435). En primer lugar, mencionaba todos aquellos registros de representaciones con guiones previamente preparados, como discursos políticos u obras dramáticas. Luego, las que son espontáneas o registradas incluso subrepticiamente y, por último, "existe un documento oral que se ha venido a llamar historia oral" y que Moss definía como "Grabación de un tipo especial de evento, la entrevista, en la que se busca deliberadamente información 
histórica, percepciones y opiniones y que se conserva deliberadamente como fuente histórica" (435).

Aun cuando en el texto de Moss la historia oral sigue siendo casi confundida con la entrevista, aunque fuera de un "tipo especial", esta empezaba a distinguirse más claramente como fuente histórica, esto es, como un documento con el cual trabaja el historiador y que tiene características particulares. En ese mismo sentido, se consideraba que debía ser complementada con otras fuentes y que había que tener particular atención de no separar los elementos que formaban parte de una misma colección, ya fuera videos, fotografías, cartas, planos u otros (De Long 164). Pese a existir consciencia acerca de las nuevas generaciones comenzaban a hablar más por teléfono y a escribir menos cartas, lo que podría afectar a los futuros historiadores; al mismo tiempo se estaba ante la paradoja de la superabundancia de registros (Benison 72). De ahí que los aspectos técnicos de la entrevista seguían siendo un elemento clave a considerar por archiveros e investigadores. Como señaló Saul Benison en 1964, "mucho se ha dicho acerca de la técnica de la historia oral, pero poco acerca de la técnica en sí, esto es, de cómo trabajan los historiadores en ella" (72).

De todos modos, mientras, como ya señalamos, Goud Colman escribió en 1965 que la historia oral había llegado "para quedarse", en 1976 Ronald Filippelli aseguró que ya no era una moda (479), en 1983 James Fogerty la calificaba como una "necesidad" (150). Detrás de ese paso desde "llegar para quedarse" a convertirse en "moda" y luego pasar a ser vista como "necesidad", existe no solo un cúmulo de experiencias en la creación, conservación y utilización de las entrevistas como fuentes orales; sino que dicha trayectoria es también reflejo de los cambios experimentados por la historiografía, en especial con la consideración de los sectores más invisibilizados de la sociedad -como mujeres, minorías étnicas y trabajadores-como sujetos históricos que requieren ser estudiados. Para la década de los setenta, los historiadores ya habían puesto su atención en aquellos grupos o individuos que suelen dejar menos registros, lo que hacía necesario levantar información inexistente en los archivos. Ello fue advertido en The American Archivist por Gerald Ham, presidente de la Society of American Archivists en su artículo "The Archival Edge" de 1975. Allí planteaba la inquietud expuesta por el historiador de la vida urbana Sam Bass Warner, quien había hecho cuatro años antes un llamado a los archiveros a hacer sus mejores esfuerzos por recopilar "un nuevo mundo de documentación material acerca de las vidas, deseos y necesidades de la gente común" (Ham 5). Algunos años más tarde, Frederic Miller nuevamente insistiría en que los archiveros debían adaptar sus prácticas a las nuevas necesidades de los historiadores, argumentando que debían comprender que los principios 
y prácticas archivísticos no eran inmutables, sino que, por el contrario, eran "producto de la comprensión de la investigación histórica de la época en que se formularon" (124).

Por otra parte, James Fogerty, de formación y trayectoria en el área de la archivística, admitía que en cualquier archivo la mayor parte de las colecciones inevitablemente tenía "vacíos" y que una manera de llenarlos era mediante la entrevista (150). Tras estas palabras escritas en 1983, hay dos cuestiones que consideramos necesario destacar. Primero, que, debido a ciertos cambios culturales y tecnológicos, los archivos y también los investigadores necesariamente se ven enfrentados a nuevos desafíos. Un ejemplo, a su parecer, estaba dado por el uso creciente del teléfono y el retroceso de las cartas en los hábitos de comunicación entre las personas. Así, este solo hecho genera vacíos en muchas colecciones, pues no dejan huellas en las vidas de muchas personas. Segundo, que dicha responsabilidad de "llenar vacíos" a través de la entrevista seguía recayendo, a su juicio, sobre los archiveros. Sin embargo, reconocía que la necesidad de complementar las colecciones a través de entrevistas - $\mathrm{O}$ "historia oral"- era más un ideal que una posibilidad real, pues "Dado que son pocos los archivos que cuentan con personal e instalaciones acordes con sus necesidades, la historia oral se ubica al final de la lista de prioridades, si es que se sitúa en ella" (153).

El hecho de que los cambios en los requerimientos de los historiadores repercutiesen en el trabajo que debían o no hacer los archiveros puso en discusión no solamente el rol de los últimos en la creación de entrevistas, sino que también en relación con el criterio que utilizaban para seleccionar y descartar material de archivo. En otras palabras, y tal como lo hicieron ver Jean-Pierre Wallot y Normand Fortier en "Archival Science and Oral Sources", los archiveros no son recolectores "neutrales" (375). En verdad, estos autores restablecieron en 1998 un viejo debate acerca de si el papel que correspondía a los archiveros era el de curadores de documentos, o creadores de los mismos (E. Swain 144). Al estar dicha discusión asociada al menos en parte a las experiencias de las entrevistas para llenar vacíos de las colecciones de archivos, o de generarlas sistemáticamente para crear colecciones; la vinculación de estas a la historia oral necesariamente introdujo con mayor fuerza a la figura del historiador dentro del debate. De hecho, el título escogido por Graham Eeles y Jill Kinner para un artículo que habían publicado diez años antes en otra revista especializada en archivística, es muy elocuente: "Archivists and Oral Historians. Friends, Strangers or Enemies?". Argumentaban que las cintas y transcripciones debían ser merecedoras de mayores cuidados en su preservación, para lo cual creían necesario un mayor trabajo colaborativo (188). 
En las décadas siguientes, los artículos dedicados a las entrevistas y a la historia oral no solo disminuyeron, sino que cambiaron de foco, pues se centraron fundamentalmente en aspectos tecnológicos, éticos y legales en relación con la creación, conservación y uso de las entrevistas como documentos de archivo (Bruemmer, 1991; E. Swain, 2003; Lawrence, 2016).

\section{ENTREVISTA Y ARCHIVO HISTÓRICO: CREACIÓN, PROPIEDAD Y USO}

Experiencias como las anteriores, ya sea que provengan de iniciativas privadas o públicas, plantean ciertos retos sobre los cuales vale la pena reflexionar, en especial para el caso de las entrevistas realizadas con fines historiográficos y/o de conservación de la memoria. En un artículo publicado en 1991, Donald A. Ritchie señalaba que, de todas las disciplinas que utilizan la entrevista como herramienta de investigación, los historiadores eran los que menos habían prestado atención metodológica a sus problemas y potencialidades (Ritchie 223). Apuntaba, asimismo, que ello contrastaba con la seriedad que dedican a las fuentes escritas. Esto podría explicarse porque, como vimos anteriormente, desde mediados del siglo XX hasta nuestros días, la tecnología disponible para hacer entrevistas fue cambiando de manera acentuada, obligando a los investigadores a adaptarse y familiarizarse con ella. Tales cambios, cabe destacar, también contribuyeron a poner en discusión la verdadera naturaleza y características epistemológicas de la entrevista, en el sentido de distinguirla como medio para obtener información, por una parte, o como producto final por otra. En palabras simples, si el historiador del tiempo presente se conformó en algún momento con las "notas" que hacía mientras el entrevistado hablaba (Donald 65; Colman 80); luego buscó la transcripción textual de la entrevista grabada como el producto esencial que había que obtener. Por último, consideró que era la grabación en sí la que, por el hecho de no solo contener palabras, sino también sonidos y la atmósfera del momento, la hacían meritoria de un estatus particular como fuente histórica, en especial cuando fue posible hacer videograbaciones (Charlton 230-231; Donald 68). Sin duda, desde la perspectiva actual, en el futuro los historiadores, así como los archiveros, deberán lidiar con las posibilidades y desafíos que la llegada de Internet ha implicado en términos de almacenamiento, selección, administración y uso de las redes sociales como fuentes.

Parte relevante de los desafíos, además de la cuestión tecnológica, deriva del hecho de que la entrevista tiene la particularidad de ser una fuente "creada" en circunstancias especiales. Como ya señalamos más arriba, en 1965 Gould Colman había escrito acerca de sus aprensiones con respecto a la fidelidad de 
la transcripción de la entrevista en relación con lo que "verdaderamente" había ocurrido entre entrevistado y entrevistador. Por esa razón, recomendaba que el último velara para que dicha transcripción fuera fiel reflejo de la entrevista (Colman 80 y 81). Dicha preocupación por la fidelidad de la transcripción radicaba en parte en que había que tener en cuenta que ni el entrevistador ni el transcriptor sabían qué usos tendría "el manuscrito que estaban creando" (Colman 82). Por ello recomendaba, como no era habitual entonces, guardar la cinta original e incluso acordar previamente las condiciones de uso con el entrevistado y la posibilidad de que revise la transcripción. Nótese que, en dicha mirada sobre la "creación" el transcriptor tomaba un rol preponderante que con el tiempo iba a perder:

El principal problema relacionado con la historia oral es cómo evitar confundir al investigador sobre lo que cada una de las tres partes involucradas en la creación de una transcripción -entrevistador, persona entrevistada y transcriptor- contribuye al producto final. (Colman 80)

Acerca del grado de participación que deben tener entrevistado y entrevistador durante la entrevista también ha habido discrepancias. Para algunos, es mejor que tome la forma de "monólogo guiado", mientras que, para otros, las preguntas y contrapreguntas, incluso las interrupciones, son no solo esenciales, sino que forman parte de la entrevista en cuanto "acto comunicativo" (De Garay 87). Como sea, el estatus de la entrevista como un documento creado por al menos dos personas $-\mathrm{y}$ normalmente por iniciativa de una de ellas- y las condiciones de uso del material resultante no solo tienen implicancias metodológicas, sino también éticas e incluso legales. Para analizar este punto, comenzaremos por el origen mismo de la entrevista, cuya naturaleza es muy particular. Como bien señala Alessandro Portelli, la entrevista no solo es "un evento en s'́,", sino además tiene una temporalidad más compleja que su duración estrictamente hablando, ya que "comienza siempre antes y termina siempre después" (Portelli 35 y 48). Con respecto a lo primero, la entrevista es un "evento" en el sentido de que es creada normalmente a iniciativa del investigador -periodista, sociólogo, historiador u otro- y no existe de manera natural. Esto significa que tras una entrevista aparece una motivación previa, que podríamos definir ampliamente como la necesidad de recabar información por parte del entrevistador acerca de temas "generales" pero, más habitualmente, sobre un tema específico circunscrito al área de interés del entrevistador. Así, y vista como una herramienta de investigación en el área de la historia, Holly Cowan agrega que no son "historias locales" sino que el 
historiador las hace como parte de un proyecto de investigación específico de manera que suelen ser hechas de acuerdo a necesidades muy particulares (Ritchie 227). Esto implica, por lo tanto, que la entrevista "comienza antes" como señala Portelli, pues requiere una preparación previa por parte de ambos, entrevistado, y en especial, entrevistador.

Al referirse a las entrevistas como "evidencias que los historiadores crean y producen", las hace diferentes de otras fuentes pues, según Cowan, las consideran su creación y por ello tiende el entrevistador a verlas como documentos personales (227). Pero argumenta que lo anterior es equivocado porque "legalmente la entrevista es de propiedad intelectual del entrevistado" (227). Esto último, como veremos más adelante, es debatible desde la perspectiva de la ley chilena. Siguiendo con la particularidad de origen de la entrevista, nos parece lúcida la apreciación que hace Portelli acerca de la naturaleza "única" de la entrevista cuando señala que "no tendremos nunca el mismo relato de una misma persona en dos momentos distintos, y mucho menos de dos personas" (Portelli 44). En otras palabras, si una misma persona puede tener recuerdos o juicios diferentes acerca de un mismo hecho en dos momentos diferentes de su vida siendo cada uno de ellos algo "único", algo similar ocurriría con una entrevista donde intervienen al menos dos sujetos. Por dicha razón, para el caso de la historia oral, "desde el momento en que el historiador comienza a entrevistar se halla a sí mismo inevitablemente trabajando con otros -al menos con sus informantes" (Thompson, "The Voice..." 27). Por ello, el entrevistador es también coautor, $y$, en consecuencia, la entrevista es un producto único hecho entre ambos.

De acuerdo con la legislación chilena, la entrevista puede ser entendida como una obra en colaboración, esto es, aquella que es producida conjuntamente por dos o más personas cuyos aportes no puedan ser separados. Entendida así, ambos, entrevistado y entrevistador, son los titulares de los derechos patrimoniales y morales que emanan de dicha obra. Aunque en cualquier entrevista es posible distinguir, al menos en apariencia, el rol de uno y otro, la obra debe ser considerada en abstracto como una unidad ya que las preguntas de uno inspiran las respuestas del otro, y estas a su vez inspiran eventualmente contrapreguntas. Así, siguiendo las reglas generales que emanan de la ley de derechos de propiedad intelectual $\left(\mathrm{n}^{\circ} 17.336\right)$, la entrevista pertenece a sus autores y respecto de ellos, la ley les confiere los derechos que emanan de su autoría por setenta años después de la muerte del último autor colaborador. En cuanto autores, estos pueden transferir los derechos patrimoniales que emanan de dicha obra a un tercero. Esta transferencia implica el derecho de este tercero a reproducir la obra de manera indefinida o estrictamente en las oportunidades 
que los autores otorgaron en su acto de cesión. También en cuanto autores, a los titulares del derecho les asiste el derecho moral de autor, que se trata de un derecho inalienable y en virtud de estos podrían, en el acto de cesión, establecer limitaciones como; mantener la obra inédita, publicarla solo a través de un seudónimo o mantenerla como anónima. Dichas limitaciones para que operen deben ser explícitamente señaladas, ya que el acto de cesión de los derechos patrimoniales implícitamente debe ser entendido como el otorgamiento a reproducir y publicar la obra por quienes accedan a ella, siempre mencionando la paternidad de la misma. Cabe señalar, sin embargo, que la ley chilena solo hace referencia especial a la entrevista periodística y a su relación con un medio de difusión.

Así, por su naturaleza y desde la perspectiva legal, la entrevista es más compleja que otras fuentes que utiliza el historiador, como cartas, periódicos e incluso diarios de vida. Involucra al menos a dos personas que, previa concertación, entablan algún tipo de relación interpersonal que puede ser mayor o menormente cercana dependiendo del contexto y técnica empleada. Puede darse de modo "impersonal", como es el caso de una entrevista por correo electrónico, con un mayor grado de contacto mediante las voces que ambos intercambian a través de un teléfono, o más cercana, como en la entrevista presencial en la que los involucrados se encuentran cara a cara, siempre habrá algún tipo de "compromiso" entre ambos, ya sea por escrito o simplemente de manera implícita. A esta relación subyacen cuestiones de poder, de ética, o lo que Portelli simplemente denomina "buenos modales". Dicho autor recuerda que en toda entrevista "estamos en el espacio y tiempo de los otros [el o los entrevistado (os)]" de manera que es responsabilidad del entrevistador generar el clima de confianza, respeto y, en ocasiones, incluso de conocer las costumbres locales a las que puede pertenecer el entrevistado. En relación a esto último, Portelli da un ejemplo extremo, el de un sociólogo asesinado en Kentucky por desconocer la diferencia cultural que existe en "entrar sin permiso a la casa" de alguien en medios urbanos y rurales (46). Agrega que estos "buenos modales" deben continuar después de la entrevista, esto es, con el uso que de ella se haga. Esto es particularmente complejo, pues ingresamos al tema del uso de la misma para los fines inicialmente concebidos por el entrevistador, que luego pueden pasar a terceros, por ejemplo, si la transcripción o grabación pasa a formar parte de un archivo. En este último caso, la "relación original" entre entrevistadorentrevistador se rompe. El mismo autor entrega el siguiente argumento: "Yo he entrevistado algunos fascistas, he hecho un libro antifascista pero me he sentido obligado a hacerlo de tal modo que los fascistas entrevistados no se sintieran insultados. Porque de algún modo me han hecho un favor" (46). 
Ese "favor", sin embargo, se diluye cuando la entrevista pasa a ser parte de un archivo en lo que Almut Leh denomina "deterioro de la relación original" (172). Cuando la entrevista es exclusivamente usada por el entrevistador en el contexto de su propia investigación, dice Leh que "no surgen problemas acerca del acuerdo alcanzado" y hasta es posible que se dé una suerte de relación de confianza entre ambos, la que "suele garantizar un trato justo y limpio respecto a lo acordado" (172). Pero la situación cambia cuando la entrevista pasa a estar a disposición de terceros, debido a que dicha relación de confianza desaparece y el entrevistado "se encuentra ahora en manos de un extraño que puede no sentirse obligado al pacto previo y a articular una interpretación mucho más crítica del protagonista" (173).

Pero el problema ético no termina aquí, pues también puede argumentarse que el investigador-entrevistador original, entonces, está "condicionado" por el favor que le hace el entrevistado de manera que pierde la libertad que cualquier trabajo científico serio debiera tener al estar teóricamente obligado a mantener esos "buenos modales" de los que hablaba Portelli. Y, en ese mismo sentido, los terceros que eventualmente pudieran trabajar con esas entrevistas conservadas en un archivo, estarían en condiciones de mayor libertad de interpretación y uso de sus contenidos. Visto desde otra perspectiva cabe preguntarse ¿qué incentivo podría tener un posible entrevistado para permitir que su entrevista sea parte de una colección de archivo si pierde totalmente las garantías de ser interpretado según lo acordado por su entrevistador, según lo que considera adecuado para que no se haga lo que él pudiera considerar "mal uso" o tergiversación de sus palabras?

Es cierto que normalmente la entrevista solo es ingresada a un archivo tras recibir el permiso explícito por parte del entrevistado y bajo condiciones que él mismo determine o apruebe. De hecho, al hacerse la entrevista debe obtenerse del entrevistado un consentimiento informado inicial y, en caso de ocurrir cambios posteriores a dicho acuerdo, este debiera renovarse. Pero esto no necesariamente resuelve todos los problemas. En el caso del archivo "Memoria Alemana" más que en el momento de la entrevista, es cuando esta pasa al archivo cuando se da el acuerdo entre ambos. Lo complejo es que, en la legislación alemana, como en las de muchas otras naciones, se entrecruzan el derecho a la información, con los de la privacidad y otros derechos. De hecho, un entrevistado que dio su consentimiento de uso de su testimonio, siempre puede retirar el permiso dado. Dicho en otras palabras, archivar entrevistas orales puede convertirse en una tarea difícil, pues implica un gran desgaste que no tiene necesariamente una recompensa cierta (Leh 173). 
Señalamos que desde que tomó fuerza la historia oral se llevaron a cabo diversas iniciativas de recolección de testimonios mediante entrevistas. A partir de entonces y en especial en las últimas décadas del siglo $\mathrm{XX}$, muchos de estos archivos se abrieron al público, generándose así un uso sistemático de dichos recursos por investigadores de diferentes disciplinas. Fue entonces cuando alcanzaron un primer plano cuestiones que hasta entonces escasamente se habían planteado, en relación con las condiciones de divulgación, restitución y transmisión de los contenidos (Descamps 3).

En el caso francés, los investigadores en humanidades y ciencias sociales que llevaron a cabo programas de recopilación de información mediante entrevistas y testimonios orales definieron sus propios dispositivos de búsqueda y protocolos de utilización, basados en la confianza y en la palabra empeñada, en el marco de la explotación científica y no comercial de dicho material (Descamps 4). Pero el tema del derecho se empezó a tratar con mayor agudeza en especial a partir de la publicación en 1990 de un manual de archivos nacionales titulado Le Témoignage aux Archives, De la collecte a la communication por Chantal de Tournier-Bonnazzi, pues la autora tocó explícitamente este punto en uno de los capítulos. La reflexión siguió en los años siguientes, en especial desde el año 2000, mediante informes, coloquios y estudios realizados por algunas organizaciones, tales como AFAS (Asociación Francesa de Archivos sonoros), la CNRS (Centre National de Recherche Scientifique), entre otras. Las conclusiones de numerosos trabajos y estudios fue, aunque parezca desilusionante, que "la situación no es simple". Como señala Florence Descamps: "Las entrevistas con fines patrimoniales o científicas tienen en efecto un estatuto complejo por el hecho de su naturaleza y de la pluralidad de actores individuales o institucionales implicados en su producción" (6). En cuanto materiales de investigación se aproximan a leyes de archivos, como creaciones originales a la ley de propiedad intelectual, si son publicados apelan al código civil, y podría seguirse. Es decir, muchos derechos se combinan. Lo mismo ocurre en el caso chileno, pues a la Ley de Propiedad Intelectual ya señalada $\left(\mathrm{n}^{\circ} 17.336\right)$, hay que agregar la Ley de Transparencia $\left(n^{\circ} 20.285\right)$ y la de Protección de la Vida Privada $\left(n^{\circ} 19.628\right)$.

Con el fin de hacerse cargo de los problemas y dudas que empezaron a surgir gracias al creciente uso de la entrevista o testimonios orales por parte de los historiadores, en mayo de 1989 la American Historical Association (AHA) acordó siete puntos que resumimos a continuación (Ritchie 236):

1. Las entrevistas deben ser grabadas solo después de que la persona a entrevistar sea informada de los derechos y responsabilidades mutuas envueltas en la historia oral (edición, confidencialidad, entre otros). 
2. El entrevistador debe esforzarse en lograr un diálogo informativo, basado en la experiencia y percepción del entrevistado y, ojalá, cotejar fuentes y datos antes de la entrevista.

3. El entrevistador debiera averiguar más allá de las necesidades inmediatas de su investigación.

4. Los entrevistadores deben ser responsables al citar sus fuentes en sus trabajos.

5. Los entrevistadores debieran gestionar el depósito de sus entrevistas en un repositorio archivístico para consultas posteriores, con todo lo que ello implica, incluso legalmente.

6. Como profesores, los historiadores deben informar a sus estudiantes acerca de las implicancias y responsabilidades al momento de entrevistar.

En otras palabras, este acuerdo no solo se hace cargo de los cuidados que debe resguardar el entrevistador en relación con el entrevistado, sino que también de las obligaciones que debe cumplir con sus estudiantes y con los investigadores futuros. Esto último, cuando señala que la entrevista debe desbordar los intereses particulares del investigador-entrevistador, además de encargarse del posterior acceso de la entrevista a través de su depósito en un archivo con todos los cuidados que ello implica. Algunos meses más tarde, la Oral History Association elaboró una guía para abordar dos grandes etapas de la entrevista en el ámbito de la historia oral: creación y uso, además de su traspaso a un archivo. Para lo último, además de asegurarse de convenir los acuerdos pertinentes con el repositorio, aconsejaba cuidar los resguardos legales necesarios en relación con el entrevistado (Ritchie 237).

Un par de años más tarde, Terry L. Birdwhistell admitía que, si bien el ideal era que las cartas de consentimiento y las transcripciones completas se integraran en los archivos; pero que en la realidad las cosas funcionaban de manera diferente: algunos conocen y adhieren a las guías de AHA u OHA; la mayoría parcialmente, no, o simplemente las desconoce (Ritchie 233). Acusaba una disparidad absoluta en el trabajo durante y posterior a la entrevista, ya que algunos transcriben todo, otros parcialmente y había quienes simplemente no lo hacían: "El punto es que la preservación de las entrevistas personales generadas por los historiadores son un camino de dos sentidos" (234). Por cada historiador que por la razón que sea no logra poner su entrevista en un archivo, hay un archivero que no quiere poner la entrevista del historiador en su archivo y transcribirla. Esto muestra que debe ser un trabajo conjunto. Pone como ejemplo la colección de historia oral de la Universidad de Kentucky (3 mil entrevistas); que al principio eran dirigidas por el staff de la biblioteca, pero con el tiempo cambió, pues fueron siendo la mayoría de las entrevistas generadas por 
“entrevistadores asociados" (historiadores, cientistas políticos, antropólogos, sociólogos. Para los casos en que las entrevistas eran hechas por estudiantes para sus proyectos, se les aseguró de no hacerlas accesibles a todo público mientras ellos no presentaran o publicaran sus trabajos (235). Consideraba que dejar esas entrevistas en un repositorio tiene múltiples ventajas: por seguridad, ya que se conserva una copia, queda en un ambiente seguro y controlado (archivo); puede ser catalogada, y eventualmente trascrita; y, lo más importante, quedarán fuera de los cajones de los historiadores donde se deteriorarían o perderían.

Evidentemente, desde esas declaraciones realizadas a fines de la década de 1980 las condiciones materiales y tecnológicas para hacer entrevistas y acceder a ellas ha cambiado sustancialmente con la masificación del uso de Internet. Es el caso de un joven que entrevistó a un veterano de la Segunda Guerra Mundial y que casi de inmediato llegó al menos a sus 700 amigos de Facebook (Ashenfelder). Es decir, esto implica que la entrevista llegue a centenares de usuarios anónimos por todo el mundo (Ashenfelder). Y ello no solo hace más compleja la arista legal a la que hemos hecho referencia más arriba, sino que, posiblemente, también interferir en el adecuado uso de su contenido debido a diferencias culturales entre sus múltiples usuarios.

\section{CONCLUSIONES}

La historia oral ha generado importantes desafíos en relación con la creación, conservación y uso del material registrado; que derivan en la necesidad de abordar su principal fuente, la entrevista, a partir de perspectivas que van desde la epistemología hasta los recursos técnicos, pasando también por cuestiones de orden ético y legal.

Las primeras experiencias realizadas en el uso sistemático de entrevista por parte de los investigadores o entidades privadas o públicas, fueron muy diferentes tanto en su origen como en sus prácticas. Algunos la emplearon para preservar la memoria de quienes vivieron hechos traumáticos, como una guerra o la violación de los derechos humanos. Otros, para no dejar en el olvido circunstancias o hechos históricos particulares, como el asesinato del presidente Kennedy o la caída del muro de Berlín. Pero, también hubo quienes simplemente han buscado rescatar la vida cotidiana de aquellas personas cuyas vidas escasamente quedan registradas en documentos archivísticos. De no hacerse tales esfuerzos, esos anónimos sujetos serían silenciados y permanecerían ausentes e invisibilizados para los futuros historiadores.

Pese a que las experiencias señaladas son diferentes tanto en sus 
objetivos y metodologías, todas ellas comparten algunos problemas, en especial aquellos de orden ético y legal desde el momento en que pasan a ser parte de un archivo para consulta pública. La propiedad conjunta de la entrevista por parte del entrevistador y el entrevistado, exige un acuerdo entre ambas partes y un consentimiento explícito. Pero, esto no significa que el entrevistado no tenga el derecho a retirar un permiso de uso, limitarlo en tiempo o a ciertas partes de la entrevista. Eso, evidentemente, genera un problema para los investigadores en general, y para el entrevistador en particular. Puede verse obligado, por ejemplo, a modificar lo ya dicho o a no utilizar lo que considere más valioso de la entrevista para los fines de su investigación. Además, el entrevistado tiene el derecho a que se preserve su privacidad, lo que genera otro escollo para el investigador. Por esta razón, algunos han optado por recurrir a "anonimizar" a su entrevistado, lo que, si bien resguarda a este último, puede representar un defecto en la calidad de la investigación ya que podría obstaculizar el cotejo de datos. Por último, recientemente se ha considerado el derecho a la privacidad, ya no solamente de las personas vivas sino también de los muertos. Sin duda en estos aspectos son muchos derechos los que se contraponen, y falta mucho por hacer en relación con la entrevista que queda en los archivos para el uso de terceros.

\section{REFERENCIAS BIBLIOGRÁFICAS}

Ashenfelder, Mike. "Doug Boyd and the Power of Digital Oral History in the 21st Century", The Library of Congress, blogs.loc.gov.

Barela, Liliana, Mercedes Miguez y Luis García Conde. Algunos apuntes sobre historia oral y cómo abordarla. Patrimonio e Instituto Histórico, Buenos Aires, 2004.

Benison, Saul. "Reflections on Oral History". The American Archivist, vol. 28, no. 1 , january 1965 , pp. 71-77.

Bonfield, Lynn A. "Conversation with Arthur M.Schlesinger, Jr.: The Use of Oral History". The American Archivist, vol. 43, no. 4, fall 1980, pp. 461472. https://doi.org/10.17723/aarc.43.4.273146611jk4367m

Bornet, Vaughn D. "Oral History Can Be Worthwhile". The American Archivist, vol. 18, no. 3, july 1955, pp. 241-253. https://doi.org/10.17723/ aarc.18.3.j583671226352285

Bruemmer, Bruce H. “Access to Oral History: A National Agenda”. The American 
Archivist, vol. 54, fall 1991, pp. 494-501. https://doi.org/10.17723/ aarc.54.4.h5828x76p28153v4

Chapoulie, Jean-Michel. La tradition sociologique de Chicago (1892-1961). Seuil, 2001. https://doi.org/10.1016/j.soctra.2003.10.025

Charlton, Myers and Rebecca Sharpless, eds. History of Oral History: Foundations and Methodology. Altamira Press, 2007.

Charlton, Thomas L. "Videotaped Oral Histories: Problemas and Prospects". The American Archivist, vol. 47, no. 3, summer 1984, pp. 228-236. https://doi.org/10.17723/aarc.47.3.35311v78u5264km4

Colman, Gould P. "Oral History-An Appeal for More Systematic Procedures". The American Archivist, vol. 28, no. 1, january 1965, pp. 79-83. https://doi.org/10.17723/aarc.28.1.e6607122380866x8

"Committee on Oral History of the Society of American Archivists". The American Archivist, vol. 36, no. 3, july 1973, pp. 361-365. https://doi.org/10.17723/aarc.36.3.244u5541t8h2066q

Danielson, Elena S. "Privacy and The Past: Research, Law, Archives, Ethics". Reviews. The American Archivist, vol. 80, no. 2, fall/Winter 2017, pp. 488-490.

Descamps, Florence. "L'Entretien de recherche en histoire: Statut juridique, contraintes et régles d'utilisation", Histoire \& Politique no. 3, nov /dic 2007, pp. 1-20. https://doi.org/10.3917/hp.003.0014

Duclert, Vincent. "Archives orales et recherche contemporaine. Une histoire en cours", Sociétés \& Représentations, vol. 13, no. 1, 2002, pp. 69-86. https://doi.org/10.3917/sr.013.0069

Eeles, Graham y Jill Kinner. "Archives and Oral Historians: Friends, Strangers or Enemies?". Journal of the Society of Archivists, october 1988, pp. 188189. https://doi.org/10.1080/00379818809511607

Filippelli, Ronald I. "Oral History and the Archives". The American Archivist, vol. 39, no. 4, october 1976, pp. 479-483. https://doi.org/10.17723/ aarc.39.4.u3kw876j8127 wl55

Fogerty, James E. "Filling the Gap: Oral History in the Archives". The American Archivist, vol. 46, no. 2, spring 1983, pp. 148-157. https://doi. org/10.17723/aarc.46.2.r775717748477g34

Ham, Gerald. "The Archival Edge". The American Archivist, vol. 38, no. 1, january 1975 , pp. 5-13.

Jullien, Dorian. "Interviews and the Historiographical Issues of Oral Sources". Till Düppe, Roy Weintraub. The Historiography of Contemporary Economics, Routledge, 2017.

Lance, David. An Archive Approach to Oral History. Imperial War Museum and 
International Association of Sound Archives, 1978.

Lathrop Gilb, Corinne. "Tape-Recorded Interviewing: Some Thoughts From California". The American Archivist, vol. 20, no. 4, october 1957. https:// doi.org/10.17723/aarc.20.4.ek22716331539362

Lawrence, Susan C. New Brunswick, N.J. Privacy and the Past: Reasearch, Law, Archives, Ethics. Rutgers University Press, 2016.

Leh, Almut."La entrevista de historia oral como material de archivo", Historia, antropología y fuentes orales, no. 21, 1999.

---. "Problems of Archiving Oral History Interviews. The Example of the Archive 'German Memory"'. FQS, vol. 1, no. 3, december 2000, Art. 8, http:// nbn-resolving.de/urn:nbn:de:0114-fqs000384.

McCann White, Helen. "Thoughts on Oral History". The American Archivist, vol. 20, no. 1, january 1957.

Michel, Henri. "Le Comité d'Histoire de la Deuxiéme Guerre Mondiale". Revue d'histoire de la Deuxième Guerre mondiale, 31e Année, no. 124, octobre 1981, pp. 1-17. https://doi.org/10.3406/ahess.1967.421614

Moss, William W. "Oral History: An Appreciation". The American Archivist, vol. 40, no. 4, october 1977, pp. 429-439. https://doi.org/10.17723/ aarc.40.4.y10q27j415278520

Neuenschwander, John. Oral History and the Law. Oxford University Press, 1993.

Nicholls, Nancy. "El desarrollo de la historia oral en Chile: de los talleres de educación popular a los estudios multidisciplinarios (1980-2013)". Historia, Voces y Memoria 6, 2013, pp. 267-302. https://doi.org/10.5944/ hme.11.2020.24179

Portelli, Alessandro. "El uso de la entrevista en la historia oral". Anuario, no. 20, Escuela de Historia, Universidad Nacional del Rosario, 2004, pp. 35-48. https://doi.org/10.14409/cya.v0i25.6920

Ritchie, Donald. A., et al. "Interviews as Historical Evidence: A Discussion of New Standards of Documentation and Access". The History Teacher, vol. 24, no. 2, february 1991, 224-237. https://doi.org/10.2307/494127

Santos, Javier. "Desarrollos metodológicos de la Escuela de Chicago", Perspectivas metodológicas, año 8, no. 8, 1965, pp. 5-.66. https://doi. org/10.18294/pm.2008.497

Swain, Donald. "Problems for Practitioners of Oral History". The American Archivist, vol. 28, no. 1, january 1965, pp. 63-69. https://doi.org/10.17723/ aarc.28.1.dk4312n1q9365x72

Swain, Ellen D. "Oral History in the Archives: Its Documentary Role in the 
Twenty-first Century". The American Archivist, vol. 66, spring/summer 2003,pp.139-158.https://doi.org/10.17723/aarc.66.1.9284q6r604858h40 Wagner Webster, Jessica. "'Filling the Gaps': Oral Histories and Underdocumented Populations in The American Archivist, 1938-2011". The American Archivist, vol.79, no. 2, fall/Winter 2016, pp. 254-282. https://doi.org/10.17723/0360-9081-79.2.254 